\title{
DIFFERENTIATING AMBIGUITY:
}

\author{
An Expository Note ${ }^{1}$ \\ Jürgen Eichberger \\ Alfred Weber Institut, \\ Universität Heidelberg, Germany. \\ David Kelsey \\ Department of Economics, \\ University of Exeter, England. \\ Simon Grant \\ Rice University, \\ Texas, USA
}

2nd September 2006

${ }^{1}$ Research supported by ESRC grant no. RES-000-22-0650. For comments and discussion we would like to thank Klaus Nehring. 


\begin{abstract}
Ghirardato, Maccheroni, and Marinacci (2004) propose a method for distinguishing between perceived ambiguity and the decision-maker's reaction to it. They study a general class of preferences which they dub invariant biseparable. This class includes CEU and MEU. This note presents some examples which illustrate their results.
\end{abstract}

Keywords, Ambiguity, multiple priors, invariant biseparable preferences, Hurwicz preferences.

JEL Classification: D81.

Address for Correspondence David Kelsey, Department of Economics, University of Exeter, Rennes Drive, Exeter, Devon, EX4 4PU, ENGLAND. 


\section{INTRODUCTION}

In a recent paper Ghirardato, Maccheroni, and Marinacci (2004) (henceforth GMM) propose a method for distinguishing between perceived ambiguity and the decisionmaker's reaction to it. GMM assume that a decision-maker deviates from expected utility if and only if there is ambiguity. In other words they attribute all departures from independence to ambiguity. This has a number of implications. Firstly they rule out other deviations from SEU such as the Allais paradox or state-dependent utility. Secondly they assume that satisfaction of the independence axiom implies the absence of ambiguity. This would be less controversial if we also assumed that the decision-maker is always averse to ambiguity. However for a decision-maker who displays both ambiguity-aversion and ambiguity-preference this second implication is not clear. Even in the presence of ambiguity, independence may be satisfied if ambiguityaversion and ambiguity-preference have equal and opposite effects on choice.

GMM show the class of preferences that they axiomatise and dub 'invariant biseparable' may be associated with a set $\mathcal{D}$ of probability distributions, which they interpret as the decision maker's perceived ambiguity, and a function $\beta(f)$ which they interpret as ambiguity attitude. These preferences may be represented in the form

$f \succcurlyeq g \Leftrightarrow \beta(f) \min _{p \in \mathcal{D}} \mathbf{E}_{p} f+(1-\beta(f)) \max _{p \in \mathcal{D}} \mathbf{E}_{p} f \geqslant \beta(g) \min _{p \in \mathcal{D}} \mathbf{E}_{p} g+(1-\beta(g)) \max _{p \in \mathcal{D}} \mathbf{E}_{p} g$,

where $\mathbf{E}_{p}$ denotes (conventional) expectation with respect to the additive probability distribution $p$ and $\beta$ is a function from the set of all acts to the unit interval. ${ }^{1}$ It is important to note that the function $\beta(f)$ depends on the act, $f$, being evaluated.

In this note we focus on the implications of GMM's results for the Choquet Expected Utility (henceforth CEU) preferences. This is the subclass of invariant biseparable preferences that can be represented by a Choquet integral with respect

\footnotetext{
${ }^{1}$ GMM refer to $\beta(f)$ as $\alpha(f)$. We have changed the notation to avoid confusion with the parameter $\alpha$ in Hurwicz preferences. As we shall show, in general, $\beta(f) \neq \alpha$ for such preferences.
} 
to a capacity. GMM show that for CEU preferences $\mathcal{D}$ is the convex hull of the decision-weights used in the Choquet integral. Since the Choquet integral of an act is the expected value of the act with respect to one of the decision-weights, its value lies between the maximum and minimum expected values of that act with respect to the decision-weights. Hence, a number $\beta(f)$ can always be defined. How useful this representation is depends on the nature of the function $\beta(f)$. We show that $\beta$ is highly variable if the dimension of $\mathcal{D}$ is greater than 2.

Although we present a few general results, the main purpose of this note is to illustrate the implications of GMM's results by studying how they apply to some common examples of CEU preferences. The first example is Hurwicz preferences, see Arrow and Hurwicz (1972) and Hurwicz (1951b), the second is the case where the set $\mathcal{D}$ consists of all convex combinations of two probability distributions.

Organisation of the paper In the next section we describe the main framework and present some general results about GMM's representation. In section 3 we introduce Hurwicz preferences and discuss how GMM's analysis applies to them. A second example where the set of priors is one dimensional is presented in section 4 and section 5 concludes.

\section{FRAMEWORK AND DEFINITIONS}

In this section we introduce the CEU model and discuss some general aspects of GMM's results. We retain GMM's assumption that the only deviations from SEU are due to ambiguity. We consider a finite set of $n$ states of nature $S$. The set of all acts is denoted by $A(S)$. For simplicity we assume that acts pay-off in utility terms. Hence an act may be represented as a function from $S$ to $\mathbb{R}$. This does not affect our analysis, which could also be expressed in terms of a conventional utility function over outcomes, if desired. 


\subsection{CEU Preferences}

We shall focus on CEU preferences, which represent a decision-maker's beliefs as capacities.

Definition 2.1 A capacity on $S$ is a real-valued function $\nu$ on the subsets of $S$ which satisfies the following properties:,

1. Monotonicity: $A \subseteq B \Rightarrow \nu(A) \leqslant \nu(B)$;

2. Normalisation: $\quad \nu(\varnothing)=0, \nu(S)=1$.

If beliefs are represented by a capacity $\nu$ on $S$, the expected utility of a given act can be found using the Choquet integral. This is defined below.

Notation 2.1 Consider a given act $f$. Let $\sigma:\{1, \ldots, n\} \rightarrow\{1, \ldots, n\}$ be a permutation such that $f\left(s_{\sigma(1)}\right) \geqslant f\left(s_{\sigma(2)}\right) \geqslant \ldots \geqslant f\left(s_{\sigma(n)}\right)$. Define a probability distribution $p^{\sigma}$ on $S$ by $p^{\sigma}\left(s_{\sigma(1)}\right)=\nu\left(s_{\sigma(1)}\right)$ and $p^{\sigma}\left(s_{\sigma(j)}\right)=\nu\left(\left\{s_{\sigma(1), \ldots,}, s_{\sigma(j)}\right\}\right)-$ $\nu\left(\left\{s_{\sigma(1), \ldots,} s_{\sigma(j-1)}\right\}\right)$ for $2 \leqslant j \leqslant n$.

Definition 2.2 The Choquet expected utility of $f$ with respect to capacity $\nu$ is:

$$
\int f d \nu=\mathbf{E}_{p^{\sigma}} f
$$

CEU is similar to subjective expected utility (henceforth SEU, see Savage (1954)), since every act is evaluated by a weighted sum of utilities. ${ }^{2}$ These weights $p^{\sigma}$ are positive and sum to 1 . The main difference from SEU, is that there are multiple sets of decision-weights and which one is used depends on the act.

Definition 2.3 For given $\nu$, let $\mathcal{D}$ denote the convex hull of the decision weights in the Choquet integral, i.e., $\mathcal{D}=\operatorname{co}\left\{p^{\sigma}: \sigma \in \Pi_{n}\right\}$, where $\Pi_{n}$ denotes the set of permutations of $\{1, \ldots, n\}$.

\footnotetext{
${ }^{2}$ Schmeidler (1989), Gilboa (1987), Sarin and Wakker (1992) and Ghirardato, Maccheroni, Marinacci, and Siniscalchi (2003) provide axioms for representing preferences by a Choquet integral of utility.
} 
GMM also show that $\mathcal{D}$ may be interpreted as a derivative, in an appropriate sense, of the preference function. However a derivative simply measures the slope of an indifference surface. Indifference surfaces in general, however, depend on both ambiguity and ambiguity-attitude. Hence it is not intuitively clear whether a derivative can separate ambiguity and ambiguity-attitude.

In general there are $n$ ! decision weights used in the Choquet integral, however these may not all be distinct. Two acts are evaluated by the same decision-weights if they are comonotonic.

Definition 2.4 Acts $f$ and $g$ are comonotonic if for all $\hat{s}, \tilde{s} \in S, f(\hat{s}) \geqslant f(\tilde{s}) \Longleftrightarrow$ $g(\hat{s}) \geqslant g(\tilde{s})$.

If acts are not comonotonic they will be evaluated by different decision-weights.

GMM use a sub-relation $\succcurlyeq^{*}$ of the decision maker's preference, which they refer to as the "unambiguous" preference.

Definition 2.5 For a CEU preference relation $\succcurlyeq$ define a sub-relation $\succcurlyeq^{*}$ by

$$
f \succcurlyeq^{*} g \Longleftrightarrow \forall p \in \mathcal{D}, \quad \mathbf{E}_{p} f \geqslant \mathbf{E}_{p} g
$$

GMM show that $\succcurlyeq^{*}$ is the maximal sub-relation of $\succcurlyeq$, which satisfies independence. (This builds on earlier work by Nehring (2001).) GMM argue that only acts which do not satisfy independence expose a decision maker to ambiguity. Therefore, in their opinion, a decision-maker deviates from SEU if and only if (s)he perceives ambiguity.

\subsection{Isotonic}

How useful the representation given by (1) is, depends on the nature of the function $\beta(f)$. In this section we establish a relationship between the variability of $\beta(f)$ and the dimension of $\mathcal{D}$. GMM show that $\beta(f)$ is constant on sets of acts which are isotonic with respect to $f$. 
Definition 2.6 Two acts $f$ and $g$ are isotonic if and only if

$$
\forall p, q \in \mathcal{D}, \mathbf{E}_{p} f \geqslant \mathbf{E}_{q} f \Rightarrow \mathbf{E}_{p} g \geqslant \mathbf{E}_{q} g
$$

GMM also show that $f$ and $g$ are isotonic if and only if there exist $\rho>0$ and $\tau$ such that,

$$
\forall p \in \mathcal{D}, \quad \mathbf{E}_{p} g=\rho \mathbf{E}_{p} f+\tau
$$

The following result shows that, in many cases, the set of acts isotonic with a given act $f$ has a smaller dimension than $A(S)$. Hence $\beta(f)$ can potentially be very variable.

Proposition 2.1 Let $f$ be a given act, then the set of acts isotonic with $f$ has dimension $n+2-r$, where $r$ is the dimension of the vector subspace spanned by $\mathcal{D}$.

Proof. We may view both acts and probabilities as vectors in $\mathbb{R}^{n}$. Expectations are then inner products, i.e., $\mathbf{E}_{p} f=p \cdot f$. With this notation, equation (2) may be rewritten:

$$
\forall p \in \mathcal{D}, \quad p \cdot(g-\rho \cdot f-\tau \cdot \mathbf{e})=0,
$$

where e denotes the unit vector in $\mathbb{R}^{n}$. Let $D$ be the vector subspace spanned by $\mathcal{D}$ and denote by $r$ the dimension of $D$. Except for SEU preferences, when $\mathcal{D}$ is a singleton set, $r \geq 2$. Equation (3) says that $g-\rho \cdot f-\tau \cdot \mathbf{e}$ lies in the orthogonal complement of $D$, which has dimension $n-r$. Since this holds for all $\rho>0$ and all $\tau$, the dimension of the space of acts isotonic with $f$ is $n+2-r$.

Thus if $r \geqslant 3$ the set of acts that are isotonic with a given act $f$ has dimension at most $n-1$ and hence is of measure zero in $\mathbb{R}^{n}$. In contrast the set of acts comonotonic with that act $f$, has dimension $n$. In this case, isotonic is a stronger restriction than comonotonic. If $\mathcal{D}$ is of full rank then equation (3) implies that the set of acts that are isotonic with a given act $f$ consists of the acts $g=\rho f+\tau e$ for arbitrary $\rho>0$ and arbitrary $\tau$. In other words, $g$ lies in a cone spanned by e and positive scalar multiples of $f$, which is a 2-dimensional subset of $\mathbb{R}^{n}$. 
As mentioned above, GMM interpret the set $\mathcal{D}$ as representing the ambiguity the decision-maker perceives and the index $\beta(f)$ as a measure of his/her attitude towards this perceived ambiguity. Proposition 2.1 shows that, for general preferences, the function $\beta(f)$ cannot be constant, if one takes the set of probability distributions derived from the partial order of independent acts $\succcurlyeq^{*}$ as the set of prior probability distributions. Note, however, that for a fixed set of prior probability distributions, assuming a constant degree of ambiguity attitude $\alpha$ does not cause any problem. As the following example of the well-known Hurwicz preferences shows one can assume that preferences of a decision-maker are characterised by constant weights $\alpha$ on the worst expected utility and $(1-\alpha)$ on the best expected utility with respect to a given set of prior probability distibutions.

The results of GMM together with Proposition 2.1 tell us however that a fixed set of priors will in general not coincide with the set of probability distributions induced by the subrelation $\succcurlyeq^{*}$. In other words, assuming that a fixed set of prior probability measures reflects the ambiguity the individual perceives there to be, and assuming the individual has a constant attitude towards this ambiguity does not correspond to the way that ambiguity and the attitude towards ambiguity is distinguished in the GMM representation.

\section{HURWICZ PREFERENCES}

For a given preference relation $\succsim$ from the GMM class of orderings, let $\mathcal{D}$ denote the set of probabilities associated with the derived preference relation $\succsim^{*}$. For each act $f$, set $\underline{f}:=\min _{P \in \mathcal{D}} \mathbf{E}_{P}[f]$ and $\bar{f}:=\max _{P \in \mathcal{D}} \mathbf{E}_{P}[f]$. Thus the GMM representation (1) may be expressed as $V(f)=\beta(f) \underline{f}+(1-\beta(f)) \bar{f}$.

For notational convenience let $\phi(i):=f\left(s_{\sigma(i)}\right)$ be the utility obtained in state $s_{\sigma(i)}$, where states are re-ordered with respect to the outcomes of $f$ from highest to lowest. If no two states have the same outcome, $\phi(i)$ is the $i$ th best outcome that 
can obtain. In particular, $\phi(1)$ is the best outcome that can arise under $f$ and $\phi(n)$ is the worst.

Consider the following class of preferences introduced by Hurwicz (1951b) and Hurwicz (1951a):

$$
f \succcurlyeq_{\alpha} g \Longleftrightarrow \alpha \min _{s \in S} f(s)+(1-\alpha) \max _{s \in S} f(s) \geqslant \alpha \min _{s \in S} g(s)+(1-\alpha) \max _{s \in S} g(s)
$$

Hurwicz introduced these preferences for situations in which the decision maker has no information about the process that will determine the state of nature. The conventional interpretation of Hurwicz preferences is that the decision maker's ambiguity is represented by the set of all probability distributions over $S, \Delta(S)$, and his/her ambiguity attitude is measured by $\alpha$ with higher values of $\alpha$ corresponding to greater ambiguity-aversion. $^{3}$

Letting $V_{a}^{H}(\cdot)$ denote this functional representation of $\succsim_{\alpha}$ in Equation (4), we have the following alternative ways to express this representation:

$$
\begin{aligned}
V_{\alpha}^{H}(f) & =\alpha \phi(n)+(1-\alpha) \phi(1) \\
& =\alpha \min _{P \in \Delta(S)} \mathbf{E}_{P}[f]+(1-\alpha) \max _{P \in \Delta(S)} \mathbf{E}_{P}[f]
\end{aligned}
$$

where $\Delta(S)$ denotes the set of probability measures on $S$ and $\mathbf{E}_{P}[f]=\sum_{i=1}^{n} P\left(s_{\sigma(1)}\right) \phi(i)$ denotes the expectation of the act $f$ with respect to the probability measure $P$. This is a special case of the well-known $\alpha$-MEU model, which has the representation

$$
V(f)=\alpha \min _{P \in \mathcal{P}} \int_{S} f(s) d P(s)+(1-\alpha) \max _{P \in \mathcal{P}} \int_{S} f(s) d P(s)
$$

for the case $\mathcal{P}=\Delta(S)$. In expression (5), the weight $\alpha$ is allocated to the least favourable probability distribution for the act, namely the (degenerate) distribution that places probability one on the state in which the worst outcome in the range of

\footnotetext{
${ }^{3}$ All the formal theories of ambiguity-aversion, which we are aware of, would agree that an increase in $\alpha$ would correspond to an increase in ambiguity-aversion, see Epstein (1999) or Ghirardato and Marinacci (2002).
} 
the act obtains (i.e. state $s_{\sigma(n)}$ on which the outcome $\phi(n)$ obtains). The remaining weight $(1-\alpha)$ is allocated to the most favourable probability distribution for this act, the (degenerate) distribution that places probability one on the state in which the best outcome in the range of the act obtains (i.e. the state $s_{\sigma(1)}$ on which the outcome $\phi(1)$ obtains).

The next result shows how GMM's representation applies to Hurwicz preferences.

Proposition 3.1 If $n \geqslant 3$, Hurwicz preferences may be represented in the form given by equation (1) provided:

1. $\mathcal{D}$ is the convex hull of the following set of $n(n-1)$ probability measures,

$$
\left\{P_{s t}^{\alpha} \in \Delta(S), s, t \in S, s \neq t: P_{s t}^{\alpha}(\{s\})=\alpha, P_{s t}^{\alpha}(\{t\})=1-\alpha, P_{s t}^{\alpha}(\{\omega\})=0, \omega \neq s, t\right\} ;
$$

2. $\beta(f)=\left\{\begin{array}{lll}\frac{\alpha-(1-\alpha)(1-\gamma)}{\alpha+(1-\alpha) \delta} & \text { if } \quad \alpha \geqslant 1 / 2, & \\ \frac{\alpha \gamma}{\alpha \delta+(1-\alpha)} & \text { if } \quad \alpha<1 / 2,\end{array}\right.$ where $\gamma=\frac{\phi(2)-\phi(n)}{\phi(1)-\phi(n)}$ and $\delta=\frac{\phi(2)-\phi(n-1)}{\phi(1)-\phi(n)}$.

Proof. Since these preferences may be represented as a Choquet integral, the form of the set $\mathcal{D}$ follows directly from GMM's Example 17, which shows the set $\mathcal{D}$ is the convex hull of the set of weights in the Choquet integral. Fix a non-constant act $f$ (that is, $\phi(1)>\phi(n)$ ). There are two cases to consider: when $\alpha$ is greater than or equal to $1 / 2$ and when $\alpha$ is less than $1 / 2$.

1. Suppose $\alpha \geqslant 1 / 2$. Then $\underline{f}=\alpha \phi(n)+(1-\alpha) \phi(n-1)$ and $\bar{f}=\alpha \phi(1)+$ $(1-\alpha) \phi(2)$. To represent the preferences in the GMM form given in (1) we require,

$$
\begin{aligned}
& \beta(f) \underline{f}+(1-\beta(f)) \bar{f} \\
= & \beta(f)[\alpha \phi(n)+(1-\alpha) \phi(n-1)]+[1-\beta(f)][\alpha \phi(1)+(1-\alpha) \phi(2)] \\
= & \alpha \phi(n)+(1-\alpha) \phi(1) .
\end{aligned}
$$


This yields

$$
\begin{aligned}
\beta(f) & =\frac{\alpha(\phi(1)-\phi(n))-(1-\alpha)(\phi(1)-\phi(2))}{\alpha(\phi(1)-\phi(n))+(1-\alpha)(\phi(2)-\phi(n-1))} \\
& =\frac{\alpha-(1-\alpha)(1-\gamma)}{\alpha+(1-\alpha) \delta} .
\end{aligned}
$$

In particular, if $\alpha=1 / 2$, then we have $\beta(f)=\gamma /(1+\delta)$.

2. Suppose $\alpha<1 / 2$. Then $\underline{f}=(1-\alpha) \phi(n)+\alpha \phi(n-1)$ and $\bar{f}=(1-\alpha) \phi(1)+$ $\alpha \phi(2)$. Now we require:

$$
\begin{aligned}
& \beta(f) \underline{f}+(1-\beta(f)) \bar{f} \\
= & \beta(f)[(1-\alpha) \phi(n)+\alpha \phi(n-1)]+[1-\beta(f)][(1-\alpha) \phi(1)+\alpha \phi(2)] \\
= & \alpha \phi(n)+(1-\alpha) \phi(1),
\end{aligned}
$$

which yields

$$
\begin{aligned}
\beta(f) & =\frac{\alpha(\phi(2)-\phi(n))}{\alpha(\phi(2)-\phi(n-1))+(1-\alpha)(\phi(1)-\phi(n))} \\
& =\frac{\alpha \gamma}{\alpha \delta+(1-\alpha)} .
\end{aligned}
$$

In figure 1 , we provide an illustration of the set $\mathcal{D}$ for the case of $n=3$ and $\alpha<1 / 2$. 
Figure 1: The set of priors $\mathcal{D}$, the convex hull of the set $\left\{P_{s t}^{\alpha} \in \Delta(S), s, t \in S, s \neq t: P_{s t}^{\alpha}(\{s\})=\alpha, P_{s t}^{\alpha}(\{t\})=1-\alpha\right\}$. for the case $n=3$ and $\alpha<1 / 2$.

In the representation given by (5) ambiguity is represented by $\Delta(S)$ and ambiguityattitude is represented by $\alpha$. In contrast GMM measure ambiguity by $\mathcal{D}$ and ambiguityattitude by $\beta(f)$. Note that $\mathcal{D}$ only coincides with $\Delta(S)$ if $\alpha=0$ or 1 . One could think that $\alpha$ is related to the ambiguity-attitude of the Hurwicz preference relation $\succsim_{\alpha}$ and yet $\mathcal{D}$ depends on $\alpha$. While $\beta(f)$ depends on $\alpha$, it also depends on $\gamma$ and $\delta$. The variable $\gamma$ is a measure of the difference between the second-best outcome and the worst outcome relative to the difference between the best and worst outcomes, while $\delta$ is a measure of the difference between the second-best and second-worst outcomes relative to the difference between the best and worst outcomes. It does not seem at all obvious to us why such variables should be relevant for an ambiguity attitude index. ${ }^{4}$

What we can also glean from the expressions for $\beta(f)$ is that $\beta(\cdot)$ is a constant

\footnotetext{
${ }^{4}$ The same utility function is used in both representations. Thus it is difficult to argue that ambiguity-attitude is somehow embedded in the utility function.
} 
function only if $\alpha=1$ or if $\alpha=0$. This means that if there are more than two states then the only Hurwicz preference relations that satisfy GMM's axiom 7 are $\succsim_{1}$ and $\succsim_{0}$, that is, the cases of extreme pessimism and extreme optimism. This is true more generally. In Eichberger, Grant, and Kelsey (2006) we show a similar result applies to all $\alpha$-MEU preferences, provided $\mathcal{D}$ consists of countably additive probability distributions.

For the case of a two-element state space, we obtain $\beta(f)=1$ if $\alpha>1 / 2$ and $\beta(f)=0$ if $\alpha<1 / 2$. For the two-element state-space, in the GMM interpretation, $\alpha$ determines the degree of perceived ambiguity which is greater the further away $\alpha$ is from $1 / 2$. The attitude towards ambiguity is dichotomous, either extreme pessimism or extreme optimism depending on whether $\alpha$ is strictly greater than or strictly less than $1 / 2$. The preference relation $\succsim_{1 / 2}$ is ambiguity neutral since it corresponds to the preference relation of an expected value maximiser who thinks each state is equally likely.

\section{ONE-DIMENSIONAL SETS OF PRIORS}

We now consider a second example where the GMM representation performs better. This is the case where $\mathcal{D}$ consists of all convex combinations of two probability distributions. Consider $\alpha$-MEU preferences of the following form.

$$
V(f)=\alpha \min _{\pi \in \mathcal{P}} \mathbf{E}_{\pi} f+(1-\alpha) \max _{\pi \in \mathcal{P}} \mathbf{E}_{\pi} f
$$

where $\mathcal{P}=\{\lambda p+(1-\lambda) q: 0 \leqslant \lambda \leqslant 1\} .^{5}$

In this case the sets of isotonic acts consist of two half spaces,

$$
H_{1}=\{f \in A(S):(p-q) \cdot f \geqslant 0\}
$$

\footnotetext{
${ }^{5}$ Whether this functional can be represented as a Choquet integral depends on the choice of $p$ and $q$.
} 
and

$$
H_{2}=\{f \in A(S):(p-q) \cdot f \leqslant 0\} .
$$

Thus $\beta(f)$ only has two possible values and is therefore much less variable than in the general case. ${ }^{6}$ In contrast, in general, there would be $2^{n}$ sets of comonotonic acts. Thus, in this case, isotonic is a less restrictive condition than comonotonic.

Without loss of generality assume that $\alpha \geqslant \frac{1}{2}$. Then these preferences have the piecewise linear form:

$$
V(f)=\left\{\begin{array}{lll}
\alpha q \cdot f+(1-\alpha) p \cdot f & \text { if } & f \in H_{1}, \\
\alpha p \cdot f+(1-\alpha) q \cdot f & \text { if } & f \in H_{2} .
\end{array}\right.
$$

The indifference surfaces have slopes equal to $\alpha q \cdot+(1-\alpha) p$ or $\alpha p+(1-\alpha) q$ hence $\mathcal{D}$ is the convex hull of $\alpha q+(1-\alpha) p$ and $\alpha p+(1-\alpha) q$. As before, the set $\mathcal{D}$ depends on $\alpha$.

If $f \in H_{1}$ then $p \cdot f \geqslant q \cdot f$, thus $\min _{\pi \in \mathcal{D}} \mathbf{E}_{\pi} f=(\alpha q+(1-\alpha) p) \cdot f$ and $\max _{\pi \in \mathcal{D}} \mathbf{E}_{\pi} f=(\alpha p+(1-\alpha) q) \cdot f$.

GMM's measure of ambiguity attitude is defined by:

$\beta(f)(\alpha q+(1-\alpha) p) \cdot f+(1-\beta(f))(\alpha p+(1-\alpha) q) \cdot f=V(f)=\alpha q \cdot f+(1-\alpha) p \cdot f$.

This implies $\beta(f)=1$ if $f \in H_{1}$. Similarly if $f \in H_{2}, \beta(f)=0$. Thus the decisionmaker either expresses extreme ambiguity-aversion or extreme ambiguity preference.

\section{CONCLUSION}

One of the main reasons that we wish to separate ambiguity and ambiguity-attitude in economic models is to conduct comparative static exercises. For instance, we may

\footnotetext{
${ }^{6}$ This is because the dimension of the subspace spanned by $\mathcal{D}$ is 2 , which we noted earlier was an exceptional case.
} 
wish to find the effect of varying ambiguity-attitude while keeping ambiguity constant. Doing such comparative static analysis GMM's framework, where ambiguity and ambiguity-attitude are simultaneously determined, is not completely straightforward.

This expository note uses some examples to illustrate the implications of GMM's axioms 1-5. In Eichberger, Grant, and Kelsey (2006) we show that the only preferences which satisfy their axioms 1-5 plus 7 are the two extreme cases MEU or maxmax expected utility.

\section{References}

Arrow, K., And L. Hurwicz (1972): "An Optimality Criterion for Decision Making under Ignorance," in Uncertainty and Expectations in Economics, ed. by C. F. Carter, and J. Ford. Blackwell, Oxford.

Eichberger, J., S. Grant, and D. Kelsey (2006): "Differentiating Ambiguity: A Comment," University of Exeter, working paper, 06/06.

Epstein, L. G. (1999): "A Definition of Uncertainty Aversion," Review of Economic Studies, 66, 579-606.

Ghirardato, P., F. Maccheroni, and M. Marinacci (2004): "Differentiating Ambiguity and Ambiguity Attitude," Journal of Economic Theory, 118, 133-173.

Ghirardato, P., F. Maccheroni, M. Marinacci, and M. Siniscalchi (2003): “A Subjective Spin on Roulette Wheels," Econometrica, 71, 1897-1908.

Ghirardato, P., and M. Marinacci (2002): "Ambiguity Made Precise: A Comparative Foundation," Journal of Economic Theory, 102, 251-289.

GilboA, I. (1987): "Expected Utility with Purely Subjective Non-additive Probabilities," Journal of Mathematical Economics, 16, 65-88. 
Hurwicz, L. (1951a): "Optimiality Criteria for Decision Making under Ignorance," Discussion paper 370, Cowles Comission.

Hurwicz, L. (1951b): "Some Specification Problems and Application to Econometric Models," Econometrica, 19, 343-344.

Nehring, K. (2001): "Decision-Making in the Context of Imprecise Probabilitistic Beliefs," working paper, University of California, Davis.

Sarin, R., And P. Wakker (1992): "A Simple Axiomatization of Non-Additive Expected Utility," Econometrica, 60, 1255-1272.

Savage, L. J. (1954): Foundations of Statistics. Wiley, New York.

Schmeidler, D. (1989): "Subjective Probability and Expected Utility without Additivity," Econometrica, 57, 571-587. 